\title{
Development of fractional order capacitors based on electrolyte processes
}

Isabel S. Jesus $\cdot$ J.A. TenreiroMachado

\begin{abstract}
In recent years, significant research in the field of electrochemistry was developed. The per- formance of electrical devices, depending on the processes of the electrolytes, was described and the physical origin of each parameter was established. However, the influence of the irregularity of the elec- trodes was not a subject of study and only recently this problem became relevant in the viewpoint of fractional calculus. This paper describes an electrolytic process in the perspective of fractional order capacitors. In this line of thought, are developed several experiments for measuring the electrical impedance of the devices. The results are analyzed through the frequency response, revealing capacitances of fractional order that can constitute an alternative to the classical integer order elements. Fractional order electric circuits are used to model and study the performance of the electrolyte processes.
\end{abstract}

\author{
Keywords \\ Fractional calculus - Electrical impedance . \\ Capacitances $\cdot$ Electrolysis
}

\section{Introduction}

Fractional calculus (FC) is a generalization of the integration and differentiation to a noninteger order. The fundamental operator is a $D_{t}^{\alpha}$, where the order $\alpha$ is a real or even a complex number, and the subscripts $a$ and $t$ represent the two limits of the operation, cf. $[1,2]$.

Recent studies brought FC into attention revealing that many physical phenomena in nature exhibit fractional behavior and can be modeled by fractional differential Eqs. [3-8].

The importance of fractional order models is that they yield a more accurate description and give a deeper insight into the physical processes underlying a long range memory behavior.

Capacitors are one of the crucial elements in integrated circuits and are used extensively in many electronic systems [9]. However, Jonscher [10] demonstrated that the ideal capacitor cannot exist in nature, because an impedance of the form $1 /(j \omega C)$ would violate causality [11, 12]. In fact, the dielectric materials exhibit a fractional behavior yielding electrical impedances of the form $1 /\left(j \omega C_{F}\right)^{\alpha}$, with $\alpha \in \mathrm{m}^{+}$[13].

Bearing these ideas in mind, this paper analyzes the fractional modeling of several electrical devices and is organized as follows. Section 2 introduces the fundamental concepts of electrical impedances. Section 3 describes the fractal geometries and fractional capacitors. Section 4 and corresponding subsections present 
the experiments results and the impedance model for the study of the fractional order capacitors. Finally, Sect. 5 draws the main conclusions.

\section{On the electrical impedance}

In an electrical circuit, the sinusoidal voltage $u(t)$ and current $i(t)$ can be expressed as a function of time $t$ :

$u(t)=U_{0} \cos (\omega t)$

$i(t)=I_{0} \cos (\omega t+\varphi)$

where $U_{0}$ and $I_{0}$ are the amplitudes of the signals, $\omega$ is the angular frequency, and $\varphi$ is the current phase shift. The voltage and current can be expressed in complex form as:

$$
\begin{aligned}
& u(t)=\operatorname{Re} U_{0} e^{j(\omega t)} \\
& i(t)=\operatorname{Re} I_{0} e^{j(\omega t+\varphi)}
\end{aligned}
$$

where $\operatorname{Re}\{\}$ represents the real part and $j={ }^{\sqrt{ }} \overline{-1}$.

Consequently, in complex form, the electrical impedance $Z(j \omega)$ is given by the expression:

$$
Z(j \omega)=\frac{\underline{U(j \omega)}}{I(j \omega)}=Z_{0} e^{j \varphi}
$$

Fractional order elements occur in several fields of engineering [9, 10, 12]. A brief reference about the Constant Phase Element (CPE) and the Warburg impedance is presented here due to their application in the work [14].

With a CPE, we have the expression:

$Z(j \omega)=\frac{1}{\left(j \omega C_{F}\right)^{\alpha}}$

where $C_{F}$ is a fractional capacitance of order $0<\alpha \leq 1$. The classical ideal capacitor occurs when $\alpha=1$.
Table 1 Impedance $Z(j \omega)$ and admittance $Y(j \omega)$ loci of $R C$ circuits of integer and fractional order

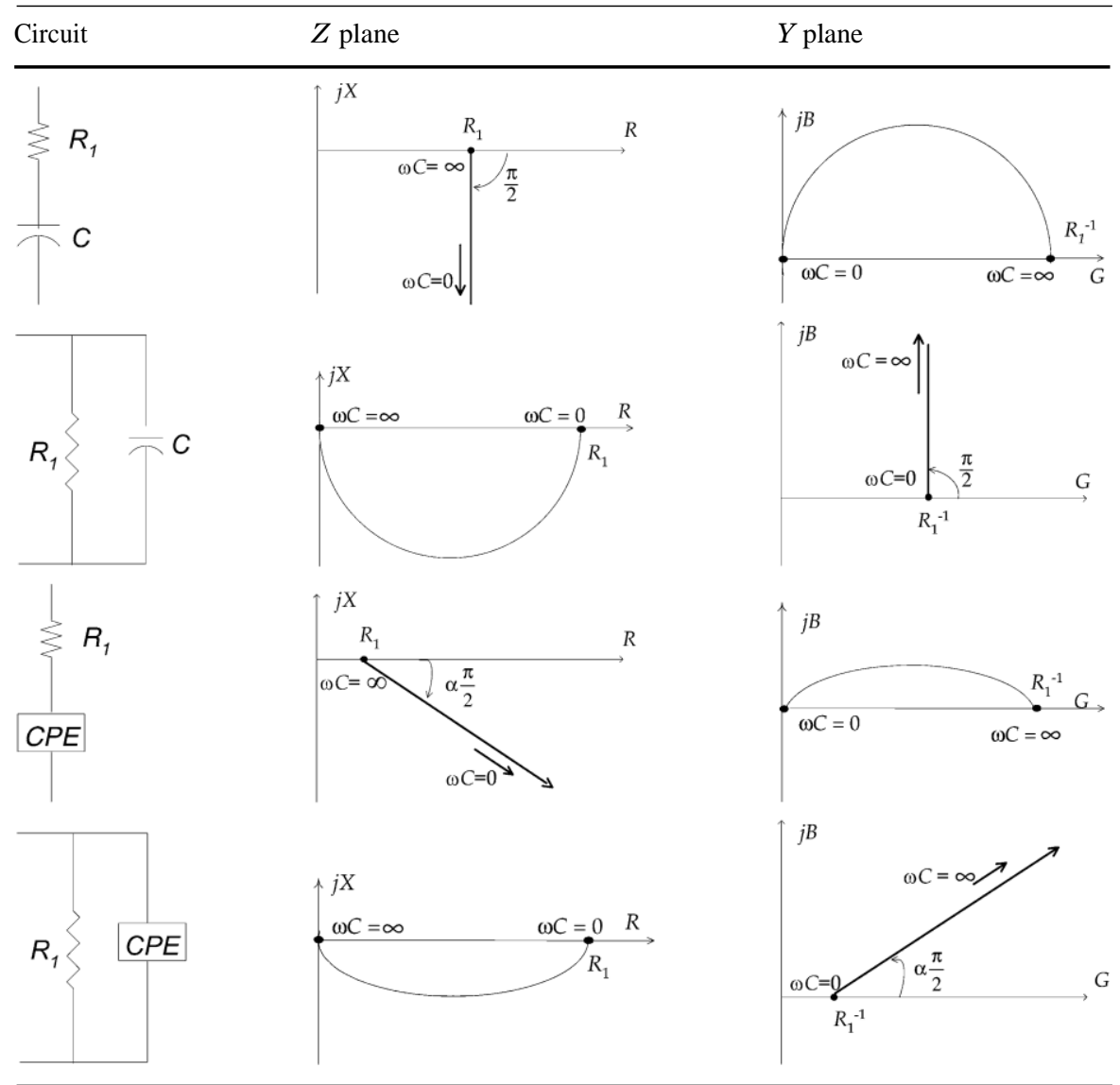


It should be noted that the SI base units of the $C_{F}$ element are $\left[\mathrm{m}^{-2 / \alpha} \mathrm{kg}^{-1 / \alpha} \mathrm{s}^{(\alpha+3) / \alpha} \mathrm{A}^{2 / \alpha}\right][15,16]$.

Table 1 shows the polar plots of the impedance $Z(j \omega)$ and the admittance $Y(j \omega)=Z^{-1}(j \omega)$ for simple series and parallel $R C$ associations of integer and fractional order, where $R=\operatorname{Re}\{Z\}$ is the resistance, $X=\operatorname{Im}\{Z\}$ is the reactance, $G=\operatorname{Re}\{Y\}$ is the conductance, and $B=\operatorname{Im}\{Y\}$ is the susceptance.

It is well known that in electrochemical systems with diffusion, the impedance is modeled by the socalled Warburg element $[14,16]$. The Warburg element arises from one-dimensional diffusion of an ionic species to the electrode. If the impedance is under an infinite diffusion layer, the Warburg impedance is given by:

$Z(j \omega)=\frac{R}{\left(j \omega C^{F}\right)^{0.5}}$

where $R$ is the diffusion resistance. If the diffusion process has finite length, the Warburg element becomes:

$Z(j \omega)=R \frac{\tanh (j \omega \tau)^{0.5}}{\tau^{0.5}}$

with $\tau=\delta^{2} / D$, where $R$ is the diffusion resistance, $\tau$ is the diffusion time constant, $\delta$ is the diffusion layer thickness, and $D$ is the diffusion coefficient $[16,17]$.

With diffusion, we have a phenomenon of fixed fractional order $\alpha=0.5$. However, several studies with fractal-like circuits [18-22], demonstrated that we can obtain other values of $\alpha$ through the recursive factors of the resistances and capacitances of the electrical network. Therefore, in this study, we try to evaluate experimentally if it is possible to merge both concepts having a diffusion phenomenon of order $0<\alpha<1$.

Based on these concepts, in the following sections, some fractional order electric impedances are presented.

\section{Fractals and fractional capacitors}

Fractals can be found both in nature and abstract objects. The impact of the fractal structures and geometries is presently recognized in engineering, physics, chemistry, economy, mathematics, art, and biology.

The concept of fractal is associated with Mandelbrot that lead to a new perception of the geometry of the nature [23]. However, the concept was initially proposed by several well-known mathematicians, such as Cantor (1872), Peano (1890), Hilbert (1891), von Koch (1904), Sierpinski (1916), Gaston (1918) and Hausdorff (1919) [23, 24].

An important geometric index consists in the fractal dimension (FDim) that represents the occupation degree in the space and that is related with its irregularity. The FDim is givenby:

$\mathrm{FDim}=\lim _{z \rightarrow \infty} \frac{\ln N(z)}{\ln (1 / \eta(z))} \approx \frac{\ln (N)}{\ln (1 / \eta)}$

where $N$ represents the number of boxes with size $\eta(N)$ resulting from the $z$ subdivisions of the original structure.

This is not the only description for the fractal geometry, but it is enough for the identification of groups with similar geometries.

Some of the classical fractals adopted in this work are the curve of Koch (FDim =1.262), carpet of Sierpinski $($ FDim $=1.893)$, curves of Hilbert $($ FDim $=$ $2.000)$, and Peano $($ FDim $=2.000)$.

The dielectric absorption in the capacitors is difficult to characterize accurately, due to the high value of the involved time constant, and the necessity of high precision measuring equipment.

The simplest capacitors are constituted by two parallel electrodes separated by a layer of insulating dielectric. There are several factors susceptive of influencing the characteristics of a capacitor. However, three of them have a special importance, namely the surface of the electrodes, the distance among them, and the material that constitutes the dielectric.

In this work, two other aspects are studied that can also influence the capacity of a capacitor, namely the wrinkling of theirs electrodes and a nonhomogenous dielectric structure. The electrodes are implemented through one-sided cooper-based printed circuit boards with the fractal geometries mentioned previously. The choice of these fractals is due to the value of FDim that it is intended to evaluate cases with a dimension from 1 up to 2 .

\section{Experimental results}

In this section, we consider three types of electrolytes, five different fractal structures, and a fractional order model of the electrical circuit. In the first subsection, 


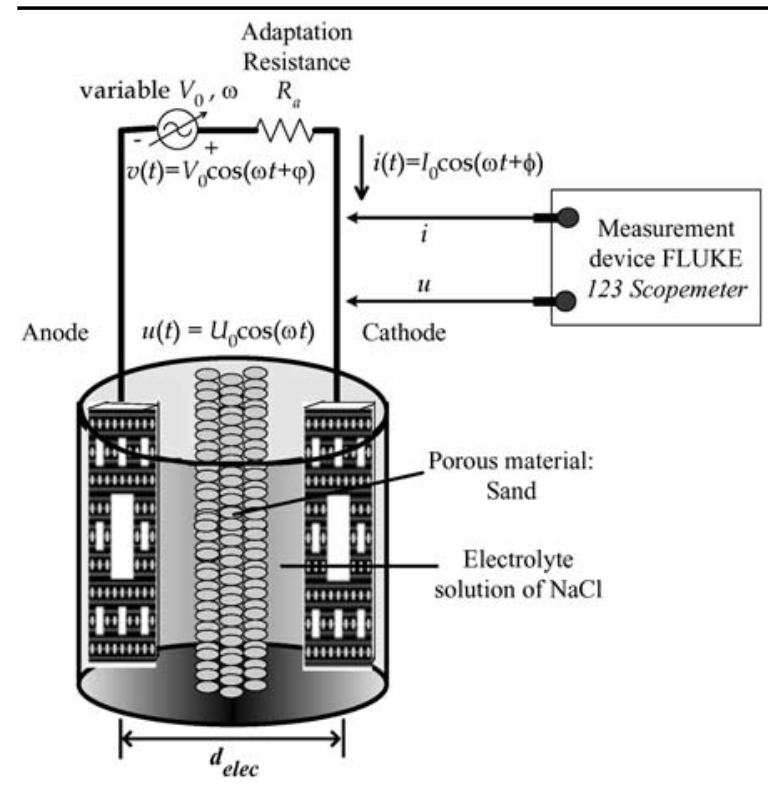

Fig. 1 Electrolyte process

we analyze the system of Fig. 1 by adopting two electrodes with the carpet of Sierpinski fractal and three different electrolytes.

In the second subsection, we adopt the approximation electrical model for analyzing several fractal electrodes and dielectric structures.

\subsection{Experiments with the carpet of Sierpinski}

In the experiments (Fig. 1) we apply sinusoidal excitation signals $v(t)$ to the apparatus, for several distinct frequencies $\omega$, and the impedance $Z(j \omega)$ between the electrodes is measured based on the resulting voltage $u(t)$ and current $i(t)$.

We study the influence of several factors such as FDim, different sodium chloride $(\mathrm{NaCl})$ solution concentrations $(\mid J J)$ and the introduction of a fractal material in the solution, namely sand. In all cases, we test also the linearity and the variation of the impedance $Z(j \omega)$ with the amplitude $V_{0}$ of the input signal.

In each experiment, we use two identical single face electrodes. The voltage, the adaptation resistance $R_{a}$, and the distance between electrodes $d_{\text {elec }}$ are kept identical during the different experiments namely, $V_{0}=10 \mathrm{~V}, R_{a}=1.2 \mathrm{k} Q$, and $d_{\text {elec }}=0.13 \mathrm{~m}$.

This methodology help us to understand the influence of the relevant factors in the impedance $Z(j \omega)$.

In a first experiment, the electrolyte process consists in a aqueous solution of $\mathrm{NaCl}$ with $\mid J J=5 \mathrm{gl}^{-1}$
(AS5) and two copper electrodes with the carpet of Sierpinski printout with an area $S=0.423 \mathrm{~m}^{2}$.

We consider two different models for describing the fractional electrical impedance. The first model consists in the resistance $R$ and $C P E$ (6) series circuit (see Table 1):

$$
Z_{a p p 1}(j \omega)=R+\overline{\left(j \omega C^{F}\right)^{\alpha}}
$$

The second model, proposed by Nigmatullin for fractional order phenomena [25, 26], consists of:

$$
\begin{aligned}
Z_{a p p 2}(j \omega)= & R_{S}+R_{c}^{r}(j \omega)^{-v}+C_{c}(j \omega)^{-v+j Q} \\
& +C_{c}{ }^{*}(j \omega)^{-v-j Q}
\end{aligned}
$$

where the symbol $*$ represents the complex conjugate.

In the first model, we have three parameters $(R$, $\left.C_{F}, \alpha\right)$ and in the second model, we have six parameters $\left(R_{s}, R_{c}, \operatorname{Re}\left\{C_{c}\right\}, \operatorname{Im}\left\{C_{c}\right\}, v, Q\right)$.

In both cases, we minimize the squared error described by:

$$
\begin{aligned}
& J_{\text {Zappi }} \\
& ={ }^{3}{ }_{k=1} \\
& \left.\mathbf{R e}\{Z\}-\operatorname{Re}\left\{Z_{a p p i}\right\}\right)^{2}+\left(\operatorname{Im}\{Z\}-\operatorname{Im}\left\{Z_{a p p i}\right\}\right)^{2}
\end{aligned}
$$

where $m$ represents the number of points and $i=1,2$. Figure 2 presents the polar diagrams of $Z(j \omega)$ and the corresponding model approximations, $Z_{a p p 1}$ and $Z_{a p p 2 .}$ For the experimental data, we get a Pear- son correlation coefficient $\rho_{Z}=-0.994$. Both mod- els

(10) and (11), reveal clearly a fractional order impedance, leading to the parameters $\left(R, C_{F}, \alpha\right)=$ (21.65, $\left.1.13 \times 10^{-4}, 0.590\right)$ and $\left(R_{s}, R_{c}, \operatorname{Re}\left\{C_{c}\right\}\right.$, $\left.\operatorname{Im}\left\{C_{c}\right\}, v, Q\right)=(19.00,179.20,0.057,0.025,0.470$, $0.770)$, and the squared error of $J_{Z a p p 1}=104.794$ and $J_{\text {Zapp2 }}=92.871$, respectively.

In a second case, with the purpose of studying the effect of the dielectric, we introduce sand into the aqueous solution of $\mid J J=5 \mathrm{gl}^{-1}$ (AS5S). We use the same electrodes and the sand completely covers the electrodes. In this case, we obtain a dielectric having also fractal characteristics. The experimental data leads to a Pearson correlation coefficient $\rho Z=-0.985$. The values of the voltage and of the adaptation resistance are identical to the previous experiment (i.e., $V_{0}=10 \mathrm{~V}, R_{a}=1.2 \mathrm{kQ}$ ). The approximation parameters yield $\left(R, C_{F}, \alpha\right)=(93.78$, 
Fig. 2 Polar diagrams of the impedance $Z(j \omega)$ and the approximations $Z_{a p p 1}(j \omega)$ and $Z_{a p p 2}(j \omega)$ for electrodes with the and the AS5 dielectric (case 1) carpet of Sierpinski fractal

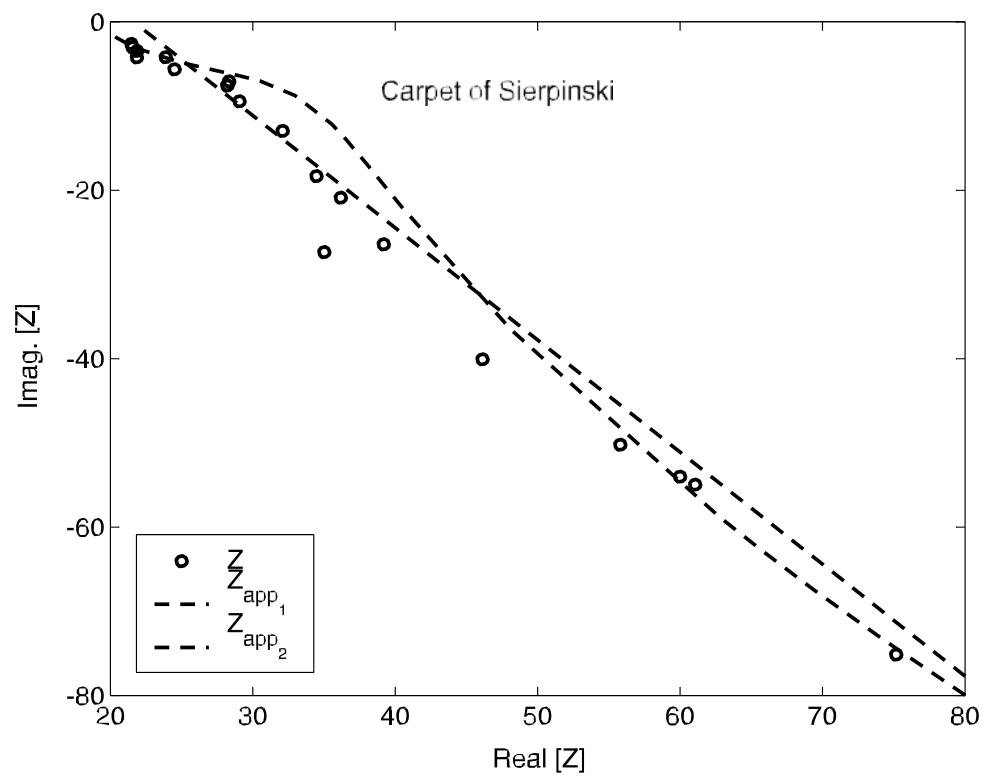

Fig. 3 Polar diagrams of the impedance $Z(j \omega)$ and the approximations $Z_{a p p 1}(j \omega)$ and $Z_{a p p 2}(j \omega)$ for electrodes with the carpet of Sierpinski fractal and the AS5 and AS5S dielectrics (cases 1 and 2)

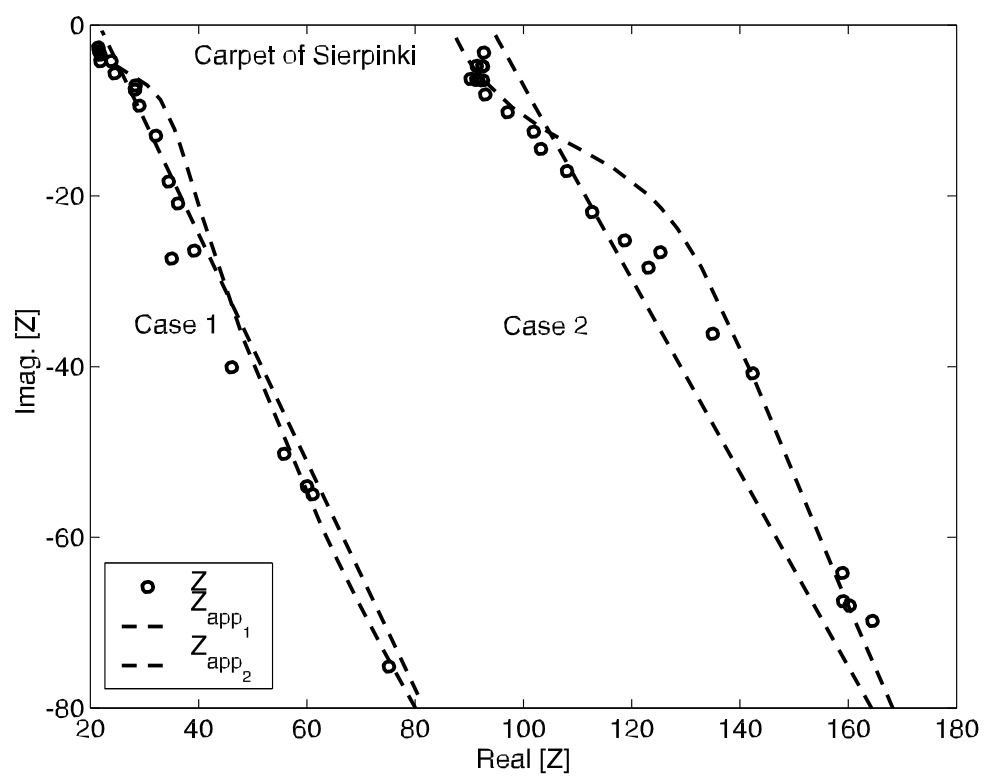

$\left.3.70 \times 10^{-5}, 0.540\right)$ and $\left(R_{s}, R_{c}, \operatorname{Re}\left\{C_{c}\right\}, \operatorname{Im}\left\{C_{c}\right\}\right.$, $v, Q)=\left(86.00,253.70,5.00 \times 10^{-8}, 0.065,0.420\right.$, 0.680 ), with squared errors of $J_{Z a p p 1}=376.417$ and $J_{\text {Zapp2 }}=303.067$, respectively.

In the two previous experiments the amplitude $V_{0}$ was varied and it was verified that the device has linear characteristics. Figure 3 illustrates the polar diagrams of $Z(j \omega)$, and the corresponding approximations, for the AS5 and AS5S dielectrics. The results reveal a good fit between the experimental data and the approximation models.

The third experiment studies the influence of the fractal surface by adopting two electrodes printed with the carpet of Sierpinski having an area of ${ }_{3} S$. The values of the voltage, the resistance of adaptation, and the solution remain unchanged (i.e., $V_{0}=$ $10 \mathrm{~V}, R_{a}=1.2 \mathrm{k} Q$ and $\left.\mid J J=5 \mathrm{gl}^{-1}\right)$. Moreover, sand was not introduced into the dielectric. The experimental data reveals a Pearson correlation coefficient 
Fig. 4 Polar diagrams of the impedance $Z(j \omega)$ and the approximations $Z_{a p p 1}(j \omega)$ and $Z_{a p p 2}(j \omega)$ for electrodes with the carpet of Sierpinski fractal, for $S$ and $\frac{1}{3} S$ and the AS5 dielectric (cases 1 and 3)

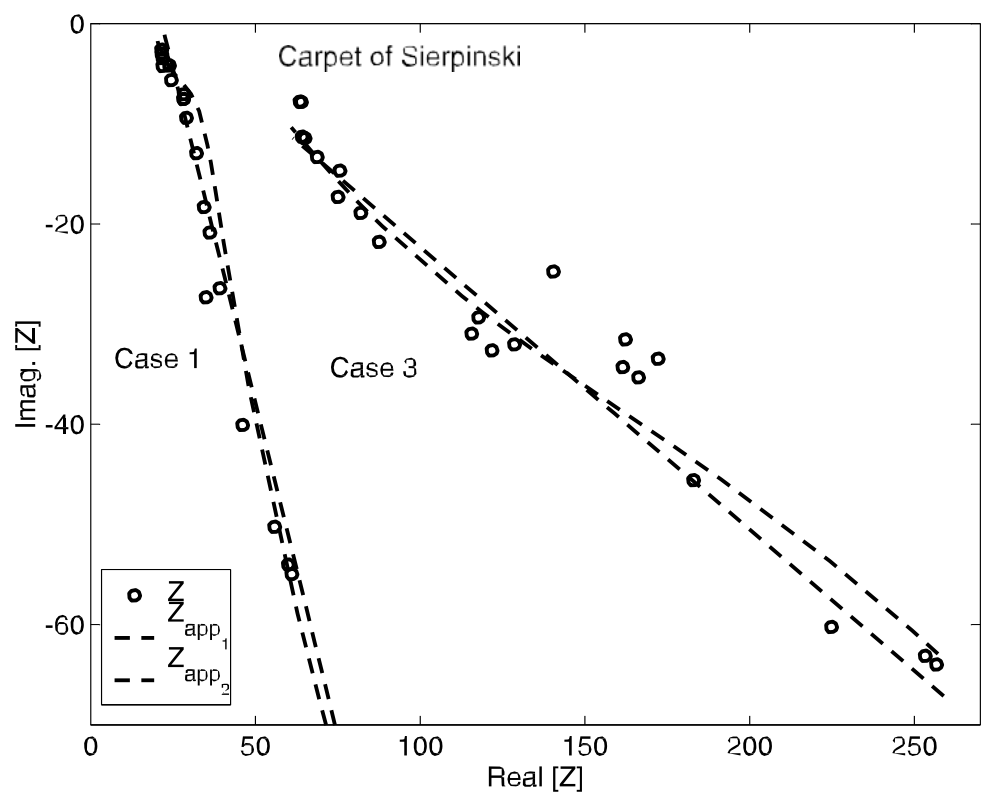

$\rho_{Z}=-0.971$. The approximation models lead to $(R$, $\left.C_{F}, \alpha\right)=\left(33.66,2.50 \times 10^{-15}, 0.200\right)$ and $\left(R_{s}, R_{c}\right.$, $\left.\operatorname{Re}\left\{C_{c}\right\}, \operatorname{Im}\left\{C_{c}\right\}, v, Q\right)=\left(29.00,784.70,6.00 \times 10^{-7}\right.$, $0.02,0.19,0.450)$, and the squared errors of $J_{Z a p p 1}=$ 157.186 and $J_{\text {Zapp } 2}=112.787$, respectively.

Figure 4 illustrates the polar diagrams of $Z(j \omega)$, and the corresponding approximations, for $S$ and $\frac{1}{3} S$ and for the AS5 dielectric. Once again, the results reveal a good fit between the experimental data and the approximation model.

In a fourth experiment, we use the same electrodes, namely the carpet of Sierpinski with an area of $\frac{1}{3} S$, but we introduce sand in the aqueous solution (AS5S). For the experimental data, we get a Pearson correlation coefficient $\rho_{Z}=-0.984$. The model parameters yield $\left(R, C_{F}, \alpha\right)=\left(195.00,8.00 \times 10^{-12}, 0.256\right)$ and $\left(R_{S}\right.$, $\left.R_{c}, \operatorname{Re}\left\{C_{c}\right\}, \operatorname{Im}\left\{C_{c}\right\}, v, Q\right)=(189.00,471.90,0.150$, $0.880,0.190,0.025)$, with squared errors of $J_{Z a p p 1}=$ 197.189 and $J_{Z a p p 2}=158.678$, respectively.

Figure 5 depicts the polar diagrams of $Z(j \omega)$, and the corresponding approximations, for $S$ and $\frac{1}{3} S$ and the AS5S dielectric.

In a fifth case, the impedance is evaluated for electrodes with the carpet of Sierpinski and area ${ }_{3}^{1} S$, but with an higher aqueous solution concentration namely of $\mid J J=10 \mathrm{gl}^{-1}$ and without sand (AS10). The voltage and the resistance of adaptation remain the same. For the experimental data, we get a Pearson correlation coefficient $\rho Z=-0.994$. The approximation leads to the parameters $\left(R, C_{F}, \alpha\right)=\left(34.15,4.00 \times 10^{-8}\right.$, $0.420)$ and $\left(R_{s}, R_{c}, \operatorname{Re}\left\{C_{c}\right\}, \operatorname{Im}\left\{C_{c}\right\}, v, Q\right)=(34.00$, $750.70,0.110,1.100,0.310,0.029)$, and squared errors of $J_{Z a p p 1}=30.568$ and $J_{Z a p p 2}=30.154$, respectively.

The results are illustrated in Fig. 6 that depicts the experimental data and the two approximations for electrodes with the carpet of Sierpinski fractal, area of $\frac{1}{3} S$, and the AS5 and AS10 dielectrics.

In a sixth experiment, the same electrodes are adopted, but sand is introduced into the aqueous solution (AS10S). For the experimental data, we get a Pearson correlation coefficient $\rho_{Z}=-0.987$. The corresponding model parameters are $\left(R, C_{F}, \alpha\right)=$ $\left(118.90,3.00 \times 10^{-9}, 0.370\right)$ and $\left(R_{s}, R_{c}, \operatorname{Re}\left\{C_{c}\right\}\right.$, $\left.\operatorname{Im}\left\{C_{c}\right\}, v, Q\right)=(104.00,676.90,0.120,1.01,0.210$, 1.31 ), with squared errors of $J_{Z a p p 1}=165.329$ and $J_{\text {Zapp } 2}=112.491$, respectively. In Fig. 7, we compare the dielectrics AS5S and AS10S for the carpet of Sierpinski with $\frac{1}{3} S$.

In this line of thought, Tables 2 and 3 summarize the values of the parameters $\left(R, C_{F}, \alpha\right)$ and $\left(R_{S}\right.$, $\left.R_{c}, \operatorname{Re}\left\{C_{c}\right\}, \operatorname{Im}\left\{C_{c}\right\}, v, Q\right)$ for the approximations $Z_{\text {app } 1}(j \omega)$ and $Z_{a p p 2}(j \omega)$, respectively, and for the six experiments described previously. We conclude that model (11) leads to a slight smaller error $J$; however, model (10) requires a smaller number of parameters that are easier to understand from the electrical viewpoint. Therefore, in the sequel, we follow the results of Table 2. 
Fig. 5 Polar diagrams of the impedance $Z(j \omega)$ and the approximations $Z_{a p p 1}(j \omega)$ and $Z_{a p p 2}(j \omega)$ for electrodes with the carpet of Sierpinski fractal, for $S$ and $\frac{1}{3} S$ and the AS5S dielectric (cases 2 and 4)

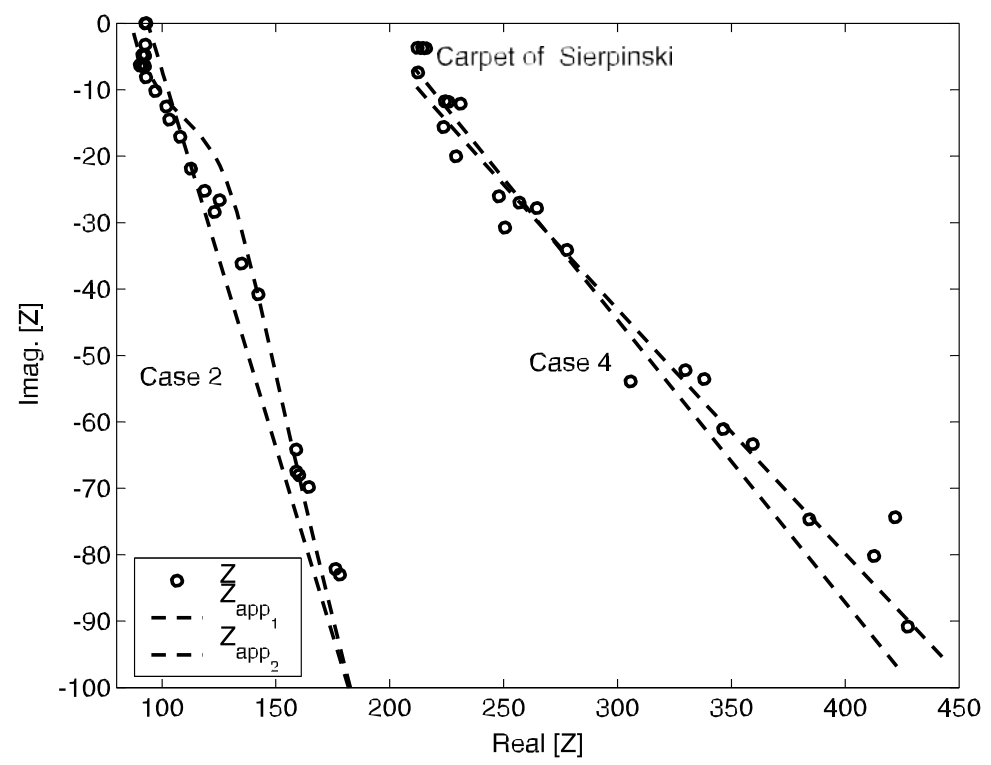

Fig. 6 Polar diagrams of the impedance $Z(j \omega)$ and the approximations $Z_{a p p 1}(j \omega)$ and $Z_{a p p 2}(j \omega)$ for electrodes with the carpet of Sierpinski fractal, for $\frac{1}{3} S$ and the AS5 and AS10 dielectrics (cases 3 and 5)

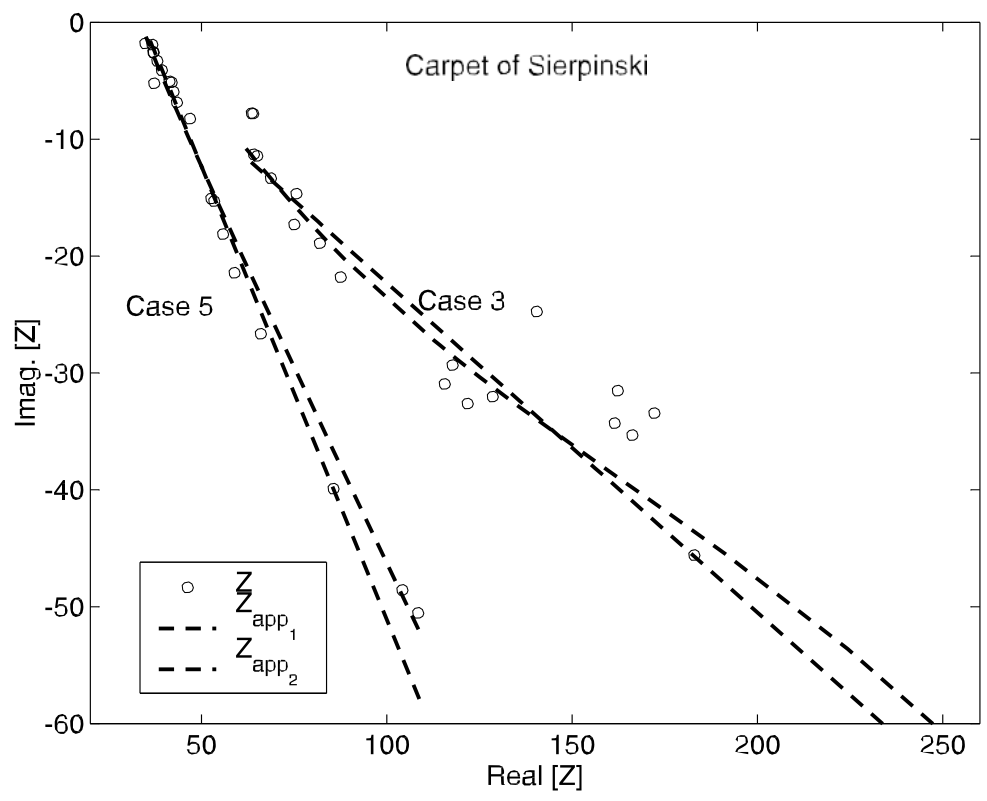

Comparing experiments 1 and 3, or 2 and 4, we conclude that decreasing the surface of the electrodes yields:

- $R_{1}<R_{3}, R_{2}<R_{4}$

$-C_{1}>C_{3}, C_{2}>C_{4}$

$-\alpha_{1}>\alpha_{3}, \alpha_{2}>\alpha_{4}$

where the indices stand for the number of the experiment.
On the other hand, comparing experiments 3 and 5, or 4 and 6 , it yields:

- $R_{3}>R_{5}, R_{4}>R_{6}$

$-C_{3}<C_{5}, C_{4}<C_{6}$

$-\alpha_{3}<\alpha_{5}, \alpha_{4}<\alpha_{6}$.

Based in the applicability of these models, in the next subsection, we organize similar experiments for the other fractals presented in Table 2 for the first 
Fig. 7 Polar diagrams of the impedance $Z(j \omega)$ and the approximations $Z_{a p p 1}(j \omega)$ and $Z_{a p p 2}(j \omega)$ for electrodes with the carpet of Sierpinski fractal, for $\frac{1}{3} S$ and the AS5S and AS10S dielectrics (cases 4 and 6)

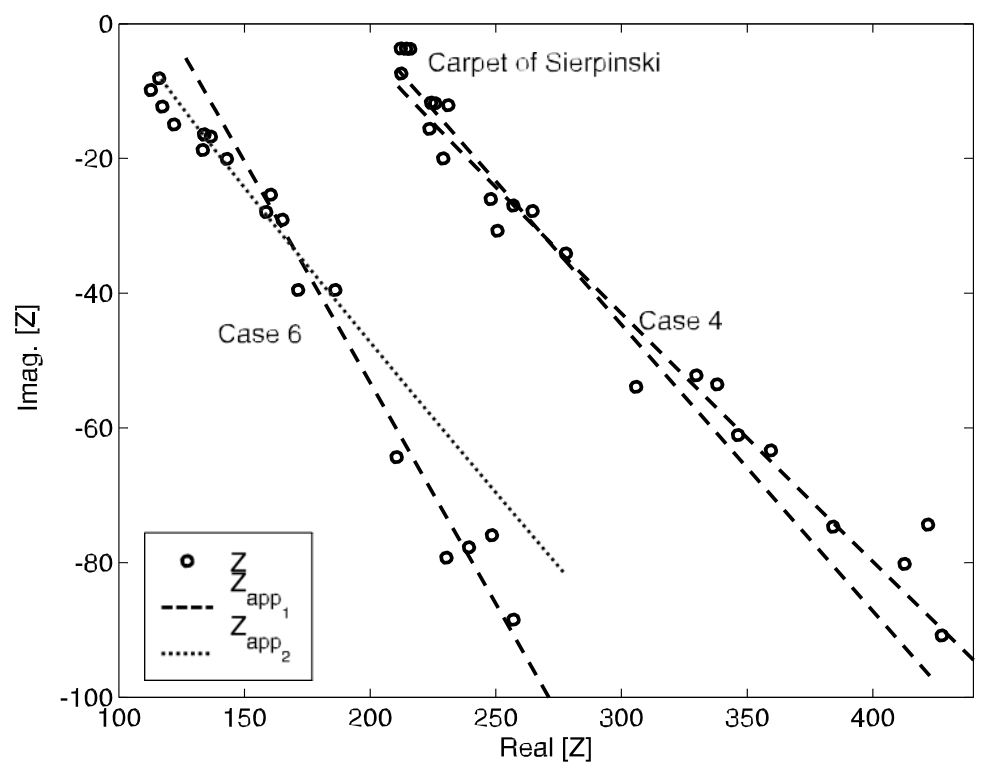

Table 2 Numerical values of the parameters for the approximation $Z_{\text {app } 1 \text {. }}$ Electrodes with the carpet of Sierpinski

\begin{tabular}{|c|c|c|c|c|c|c|c|}
\hline Case & Fractal & $\begin{array}{l}\text { Sur- } \\
\text { face }\end{array}$ & |JJ & $\begin{array}{l}R \\
{[Q]}\end{array}$ & $\begin{array}{l}C_{F} \\
{\left[\mathrm{~m}^{-2 / \alpha} \mathrm{kg}^{-1 / \alpha}\right.} \\
\left.\mathrm{s}^{(\alpha+3) / \alpha} \mathrm{A}^{2 / \alpha}\right]\end{array}$ & $\alpha$ & $J$ \\
\hline 1 & $\begin{array}{l}\text { Carpet of } \\
\text { Sierpinski }\end{array}$ & $S$ & AS5 & 21.65 & $1.13 \times 10^{-4}$ & 0.590 & 104.794 \\
\hline 2 & $\begin{array}{l}\text { Carpet of } \\
\text { Sierpinski }\end{array}$ & $S$ & AS5S & 93.78 & $3.70 \times 10^{-5}$ & 0.540 & 376.417 \\
\hline 3 & $\begin{array}{l}\text { Carpet of } \\
\text { Sierpinski }\end{array}$ & $\frac{1}{3} S$ & AS5 & 33.66 & $2.50 \times 10^{-15}$ & 0.200 & 157.186 \\
\hline 4 & $\begin{array}{l}\text { Carpet of } \\
\text { Sierpinski }\end{array}$ & $\frac{1}{3} S$ & AS5S & 195.00 & $8.00 \times 10^{-12}$ & 0.256 & 197.188 \\
\hline 5 & $\begin{array}{l}\text { Carpet of } \\
\text { Sierpinski }\end{array}$ & $\frac{1}{3} S$ & AS10 & 34.15 & $4.00 \times 10^{-8}$ & 0.420 & 30.569 \\
\hline 6 & $\begin{array}{l}\text { Carpet of } \\
\text { Sierpinski }\end{array}$ & $\frac{1}{3} S$ & AS10S & 118.90 & $3.00 \times 10^{-9}$ & 0.370 & 165.329 \\
\hline
\end{tabular}

model $\left(Z_{a p p 1}\right)$ in order to analyze their influence upon the electrical impedance.

\subsection{Experiments with other fractals}

This subsection analyzes the influence of the fractal printed in the surface of the electrode. In this line of thought, the adoption of the curves of Koch, Hilbert, and Peano is studied.
The values of the voltage amplitude $V_{0}$, the resistance of adaptation $R_{a}$, and the dielectric solution (AS5) remain identical to those considered in the previous experiments. The size of the fractals was adjusted so that their surface yields identical values, namely $S=0.423 \mathrm{~m}^{2}$. In all experiments, the linear characteristics of the device were confirmed again. The behaviors for dielectrics were also investigated without and with sand as described previously for the 
Table 3 Numerical values of the parameters for the approximation $Z_{a p p 2}$. Electrodes with the carpet of Sierpinski

\begin{tabular}{llllllllr}
\hline Case & $\mid J J$ & \multicolumn{1}{l}{$R_{s}$} & $\begin{array}{l}R_{c} \\
{[Q]}\end{array}$ & $\operatorname{Re}\left\{C_{c}\right\}$ & $\operatorname{Im}\left\{C_{c}\right\}$ & $v$ & $Q$ & $J$ \\
\hline 1 & AS5 & 19.00 & 179.20 & 0.057 & 0.025 & 0.470 & 0.770 & 92.871 \\
2 & AS5S & 86.00 & 253.70 & $5.0 \times 10^{-8}$ & 0.065 & 0.420 & 0.680 & 303.067 \\
3 & AS5 & 29.00 & 784.70 & $6.0 \times 10^{-7}$ & 0.020 & 0.190 & 0.450 & 112.787 \\
4 & AS5S & 189.00 & 471.90 & 0.150 & 0.880 & 0.190 & 0.025 & 158.678 \\
5 & AS10 & 34.00 & 750.70 & 0.110 & 1.100 & 0.310 & 0.029 & 30.154 \\
6 & AS10S & 104.00 & 676.90 & 0.120 & 1.010 & 0.210 & 0.031 & 112.491 \\
\hline
\end{tabular}

Table 4 Numerical values of the parameters of the circuit with $R$ and $\mathrm{CPE}$ series association for several fractal electrodes

\begin{tabular}{|c|c|c|c|c|c|c|c|}
\hline Case & Fractal & Surface & $\mid J J$ & $\begin{array}{l}R \\
{[Q]}\end{array}$ & $\begin{array}{l}C_{F} \\
{\left[\mathrm{~m}^{-2 / \alpha} \mathrm{kg}^{-1 / \alpha}\right.} \\
\left.\mathrm{s}^{(\alpha+3) / \alpha} \mathrm{A}^{2 / \alpha}\right]\end{array}$ & $\alpha$ & $J$ \\
\hline 7 & $\begin{array}{l}\text { Curve of } \\
\text { Koch }\end{array}$ & $S$ & AS5 & 22.72 & $7.30 \times 10^{-5}$ & 0.602 & 156.640 \\
\hline 8 & $\begin{array}{l}\text { Curve of } \\
\text { Hilbert }\end{array}$ & $S$ & AS5 & 20.67 & $1.39 \times 10^{-4}$ & 0.659 & 213.319 \\
\hline 9 & $\begin{array}{l}\text { Curve of } \\
\text { Peano }\end{array}$ & $S$ & AS5 & 20.39 & $4.30 \times 10^{-5}$ & 0.540 & 99.391 \\
\hline 10 & $\begin{array}{l}\text { Curve of } \\
\text { Koch }\end{array}$ & $S$ & AS5S & 92.81 & $6.50 \times 10^{-5}$ & 0.610 & 235.826 \\
\hline 11 & $\begin{array}{l}\text { Curve of } \\
\text { Hilbert }\end{array}$ & $S$ & AS5S & 78.97 & $1.80 \times 10^{-4}$ & 0.700 & 211.101 \\
\hline 12 & $\begin{array}{l}\text { Curve of } \\
\text { Peano }\end{array}$ & $S$ & AS5S & 95.80 & $4.50 \times 10^{-5}$ & 0.580 & 256.485 \\
\hline
\end{tabular}

carpet of Sierpinski. These experiments constitute the cases 7-12.

Table 4 shows the values of the parameters $\left(R, C_{F}\right.$, $\alpha)$ and Fig. 8 depicts the polar diagrams of $Z(j \omega)$ and $Z_{a p p}(j \omega)$.

These figures reveal similarities with the results presented in the Table 1. Comparing experiments 1 , 7,8 , and 9 with $2,10,11$, and 12 , we conclude that the introduction of sand in the solution leads to:

- $R_{1}<R_{2}, R_{7}<R_{10}, R_{8}<R_{11}, R_{9}<R_{12}$

- $C_{1} \approx C_{2}, C_{7} \approx C_{10}, C_{8} \approx C_{11}, C_{9} \approx C_{12}$

$-\alpha_{1} \approx \alpha_{2}, \alpha_{7} \approx \alpha_{10}, \alpha_{8} \approx \alpha_{11}, \alpha_{9} \approx \alpha_{12}$.

The comparison of all experiments shows that only the evolution of $R$ is straightforward, namely that it increases with the fractional order of the dielectric and that decreases with the solution concentration $\mid J J$.
The parameters $\alpha$ and $C_{F}$ increase when $S$ increases, when the solution concentration $\mid J J$ increases, and remain approximately constant for different dielectric structures. Nevertheless, in order to better quantify this behavior, further research is needed, namely the development of other experiments with other values of $S$.

An important aspect not considered in this paper is the 3D wrinkling of the electrodes and, consequently, new experiments should also evaluate that possibility.

In what concerns the model provided by $Z_{a p p}$, it is clear that adopting more complex circuits, we can have better approximations. Nevertheless, models with a larger number of elements make difficult the comparison of the different cases and also to assign a clear and simple physical meaning to each parameter. Therefore, in the authors opinion, further research should focus mainly on the electrode physical design. 


\section{Conclusions}
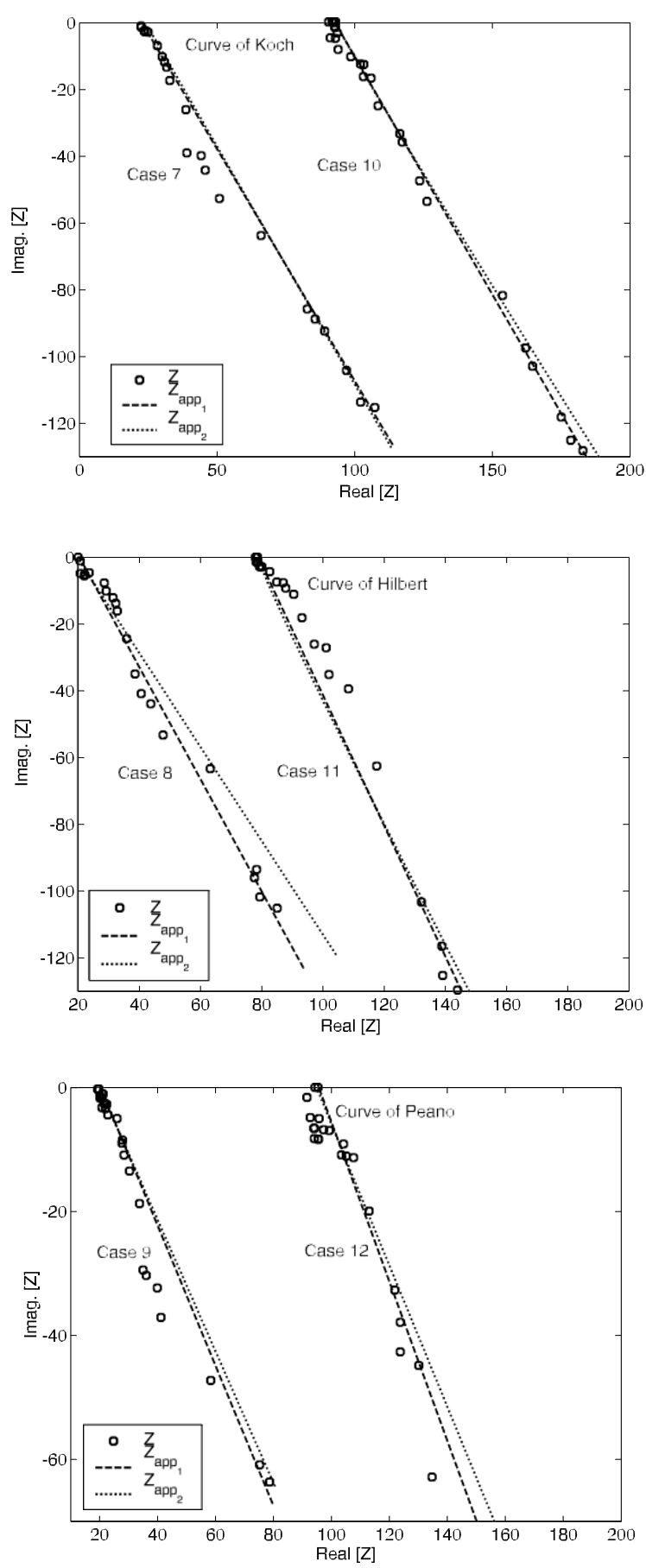

Fig. 8 Polar diagrams of the impedance $Z(j \omega)$, the approximations $Z_{a p p 1}(j \omega)$ and $Z_{a p p 2}(j \omega)$ and for the fractal electrodes $\{$ Koch, Hilbert and Peano\} and the AS5 and AS5S dielectrics (cases 7 and 10, 8 and 11, and 9 and 12, respectively)
During several centuries, the FC was developed mainly in a mathematical viewpoint, but presently it addresses a considerable range of applications. In this paper, the FC concepts were applied in the modeling of electrical fractional impedances. Therefore, fractal structures were adopted in an electrolyte process. This system is a possible prototype for the development of fractional electrical devices, and may be considered as an alternative to the classical integer order capacitors. The experimental results demonstrate that is possible to get fractional order elements by adopting nonclassical electrodes.

\section{References}

1. Oldham, K.B., Spanier, J.: The Fractional Calculus: Theory and Application of Differentiation and Integration to Arbitrary Order. Academic Press, New York (1974)

2. Miller, K.S., Ross, B.: Introduction to the Fractional Calculus and Fractional Differential Equations. Wiley, New York (1993)

3. Samko, S.G., Kilbas, A.A., Marichev, O.I.: Fractional Integrals and Derivatives: Theory and Applications. Gordon and Breach, New York (1993)

4. Debnath, L.: In: 41st IEEE Conference. Fractional Calculus and Its Applications, November 2002

5. Tenreiro Machado, J.A., Jesus, I.S.: A suggestion from the past?. J. Fract. Calc. Appl. Anal. 7(4), 403-407 (2005)

6. Jesus, I.S., Tenreiro Machado, J.A., Cunha, J.B., Silva, M.F.: Fractional order electrical impedance of fruits and vegetables. In: Proc. 25th IASTED International Conference on Modeling, Identification and Control-MIC06, pp. 489-494, Spain, February 6-8 2006

7. Nigmatullin, R.R., Alekhin, A.P.: Quasi-fractals: new method of description of a structure of disordered media. In: 2nd IFAC Workshop on Fractional Differentiation and Its Applications-FDA06, Porto, Portugal, 19-21 July 2006

8. Nigmatullin, R.R., Arbuzov, A.A., Salehli, F., Giz, A., Bayrak, I., Catalgil-Giz, H.: The first experimental confirmation of the fractional kinetics containing the complexpower-law exponents: Dielectric measurements of polymerization reactions. Physica B 388, 418-434 (2007)

9. Samavati, H., Hajimiri, A., Shahani, A.R., Nasserbakht, G.N., Lee, T.H.: Fractal capacitors. IEEE J. Solid-State Circuits 33(12), 2035-2041 (1998)

10. Jonscher, A.K.: Dielectric Relaxation in Solids. Chelsea Dielectric Press, London (1993)

11. Bohannan, G.W.: Analog realization of a fractional order control element. Wavelength Electronics (2002)

12. Bohannan, G.W.: Interpretation of complex permittivity in pure and mixed crystals. Wavelength Electronics (2002)

13. Westerlund, S.: Capacitor theory, IEEE Trans. Dielectr. Electr. Insul. 1(5), 826-839 (1994) 
14. Barsoukov, E., Macdonald, J.R.: Impedance Spectroscopy, Theory, Experiment, and Applications. Wiley, New York (2005)

15. Jesus, I.S., Tenreiro Machado, J.A., Cunha, J.B.: Fractional electrical dynamics in fruits and vegetables. In: 2nd IFAC Workshop on Fractional Differentiation and its Applications, Porto, Portugal, 19-21 July 2006

16. Jesus, I.S., Tenreiro Machado, J.A., Cunha, J.B.: Fractional electrical impedances in botanical elements, J. Vibr. Control (2008, in press)

17. Jesus, I.S., Tenreiro Machado, J.A., Silva, M.F.: Fractional order capacitors. In: Proc. 27th IASTED International Conference on Modeling, Identification and Control, Austria, February 11-13 2008

18. Clerc, J.P., Tremblay, A.-M.S., Albinet, G., Mitescu, C.D.: A.c. response of a fractal networks. J. Phys. Lett. 45(19), L913-L924 (1984)

19. Kaplan, T., Gray, L.J.: Effect of disorder on a fractal model for the ac response of a rough interface. J. Phys. B 32(11), 7360-7366 (1985)
20. Liu, S.H.: Fractal model for the ac response of a rough interface. J. Phys. 55(5), 529-532 (1985)

21. Kaplan, T., Liu, S.H., Gray, L.J.: Inverse-Cantor-bar model for the ac response of a rough interface. J. Phys. B 34(7), 4870-4873 (1986)

22. Kaplan, T., Gray, L.J., Liu, S.H.: Self-affine fractal model for a metal-electrolyte interface. J. Phys. B 35(10), 5379 5381 (1987)

23. Falconer, K.: Fractal Geometry-Mathematical Foundation and Applications. Wiley, New York (1990)

24. Mehaute, A.L., Fractal Geometries: Theory and Applications, Penton Press, London (1990)

25. Nigmatullin, R.R., Le Mehaute, A.: The geometrical and physical meaning of the fractional integral with complex exponent. Int. J. Sci. Georesources 1(8), 2-9 (2004)

26. Nigmatullin, R.R., Le Mehaute, A.: Is there geometri$\mathrm{cal} /$ physical meaning of the fractional integral with complex exponent? J. Non-Cryst. Solids 351, 2888-2899 (2005) 